21st Particles and Nuclei International Conference (PANIC 2017)

International Journal of Modern Physics: Conference Series

Vol. 46 (2018) 1860003 (8 pages)

(C) The Author(s)

DOI: $10.1142 / \mathrm{S} 2010194518600030$

\title{
Nuclear Astrophysics Deep Underground
}

\author{
Rosanna Depalo \\ Dipartimento di Fisica e Astronomia, \\ Universitá degli Studi di Padova and INFN Padova \\ Via Marzolo, 8 Padova, 35131, Italy \\ rdepalo@pd.infn.it
}

Published 3 May 2018

\begin{abstract}
Cross sections of nuclear reactions relevant for astrophysics are crucial ingredients to understand the energy generation inside stars and the synthesis of the elements. At astrophysical energies, nuclear cross sections are often too small to be measured in laboratories on the Earth surface, where the signal would be overwhelmed by the cosmic-ray induced background.

LUNA is a unique Nuclear Astrophysics experiment located at Gran Sasso National Laboratories. The extremely low background achieved at LUNA allows to measure nuclear cross sections directly at the energies of astrophysical interest. Over the years, many crucial reactions involved in stellar hydrogen burning as well as Big Bang nucleosynthesis have been measured at LUNA.

The present contribution provides an overview on underground Nuclear Astrophysics as well as the latest results and future perspectives of the LUNA experiment.
\end{abstract}

Keywords: Nuclear Astrophysics; Underground laboratories; Nucleosynthesis.

\section{Introduction}

Nuclear fusion reactions provide most of the energy radiated by stars. Indeed, the whole life of a star consists of a sequence of phases in which heavier and heavier elements are burnt inside the stellar core. Moreover, nuclear reactions are responsible for the synthesis of the elements during the Big Bang and inside stars.

Theoretical models have been developed to try to reproduce the elemental abundances observed in the stellar surface. An crucial input for these models is the thermonuclear reaction rate for all the nuclear reactions involved.

At astrophysical temperatures, the kinetic energy of the interacting particles follows a Maxwell-Boltzmann distribution and the average energy is usually much lower than the Coulomb repulsive potential between the nuclei. Therefore, nuclear

This is an Open Access article published by World Scientific Publishing Company. It is distributed under the terms of the Creative Commons Attribution 4.0 (CC-BY) License. Further distribution of this work is permitted, provided the original work is properly cited. 


\section{R. Depalo}

reactions can only occur by quantum mechanical tunneling and the cross section decreases exponentially with the energy ${ }^{1}$.

As a consequence of the interplay between the energy distribution of nuclei and the tunneling probability through the Coulomb barrier, thermonuclear reactions can only occur in a well defined energy range, called the Gamow peak. At Gamow energies, nuclear cross sections become extremely small (of the order of $10^{-9}-10^{-12}$ barn), therefore, in typical experimental conditions, the expected counting rate can be much smaller than the environmental background in a detector.

Environmental background is produced by cosmic rays and the decay of naturally-occurring radioactive isotopes (uranium and thorium chains and ${ }^{40} \mathrm{~K}$ ). The background from radioactive isotopes can be substantially reduced by shielding the detector with high $\mathrm{Z}$ and high density material (usually lead or copper). On the other hand, cosmic radiation at sea level is mainly made of muons, highly penetrating particles created in the upper atmosphere that leave a trace in most particle and gamma ray detectors and can generate spallation reactions in detector and surrounding materials, with consequent production of neutrons and radioactive nuclei. The most efficient way to suppress the muon-induced background is to perform experiments in deep-underground laboratories ${ }^{2,3}$.

\section{The LUNA experiment}

LUNA (Laboratory for Underground Nuclear Astrophysics) is located at the Gran Sasso underground laboratories, in central Italy. The laboratory is shielded by 1400 meters of mountain rocks, attenuating the cosmic-ray muon flux by about six orders of magnitude when compared with the surface of the Earth.

The LUNA laboratory was started in 1991 with the installation of a $50 \mathrm{kV}$ accelerator $^{4}$. In 2001 the $50 \mathrm{kV}$ machine was replaced by a $400 \mathrm{kV}$ accelerator, which is still operational today ${ }^{5}$. A third and more energetic accelerator, named LUNA MV, will be installed in $2018^{6,7,8,9}$.

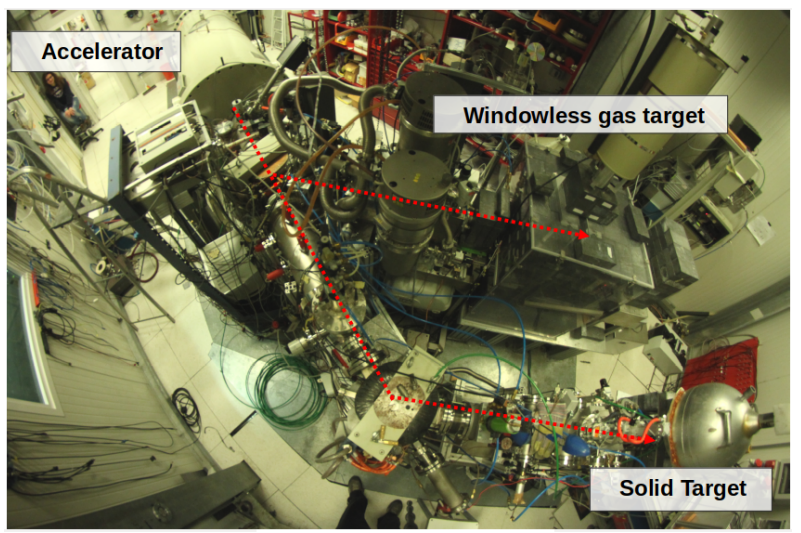

Fig. 1. Photograph of the LUNA - $400 \mathrm{kV}$ experimental hall. 
The LUNA experiment is presently equipped with a $400 \mathrm{kV}$ electrostatic accelerator with a radio-frequency ion source. The accelerator provides proton or alpha beams with intensity as high as $500 \mu \mathrm{A}$ on target. The high voltage is generated by an inline Cockcroft-Walton power supply. The beam energy stability is particularly important for nuclear astrophysics experiments, since the fusion cross section below the Coulomb barrier depends exponentially on the energy. The LUNA proton beam energy is calibrated with $0.3 \mathrm{keV}$ accuracy and has a long term stability of $5 \mathrm{eV} / \mathrm{h}$. The beam energy spread has been measured to be about $100 \mathrm{eV}^{5}$. The ion beam can be delivered either to a solid target or to a windowless gas target. Different gamma-ray or particle detectors can be used, depending on the characteristics of the nuclear reaction to be studied.

The LUNA setup is ideally suited for the study of hydrostatic hydrogen burning taking place in stars at temperatures of $0.01-0.1 \mathrm{GK}^{10}$ and also explosive burning at temperatures up to $1 \mathrm{GK}$ in sites such as the Big Bang, classical novae and supernovae $^{11,12}$.

Over the years, a large number of reactions involved in stellar hydrogen burning and Big Bang Nucleosynthesis have been investigated at LUNA ${ }^{9}$. Fig. 2 provides an overview of all reactions of the pp chain and the CNO cycle which have been measured at LUNA. The cross section of those reactions was measured either directly in the Gamow window or very close to it.

\section{Latest results}

This sections provides a short review of some of the most recent results published by the LUNA collaboration, as well as a brief description of the experiments presently ongoing at the $400 \mathrm{kV}$ accelerator and future perspectives.

\subsection{The ${ }^{17} O(p, \alpha)^{14} N$ reaction}

In novae explosions and Asymptotic Giant Branch (AGB) stars, hydrogen burning proceeds mainly through the $\mathrm{CNO}$ cycle. Two key reactions of the $\mathrm{CNO}$
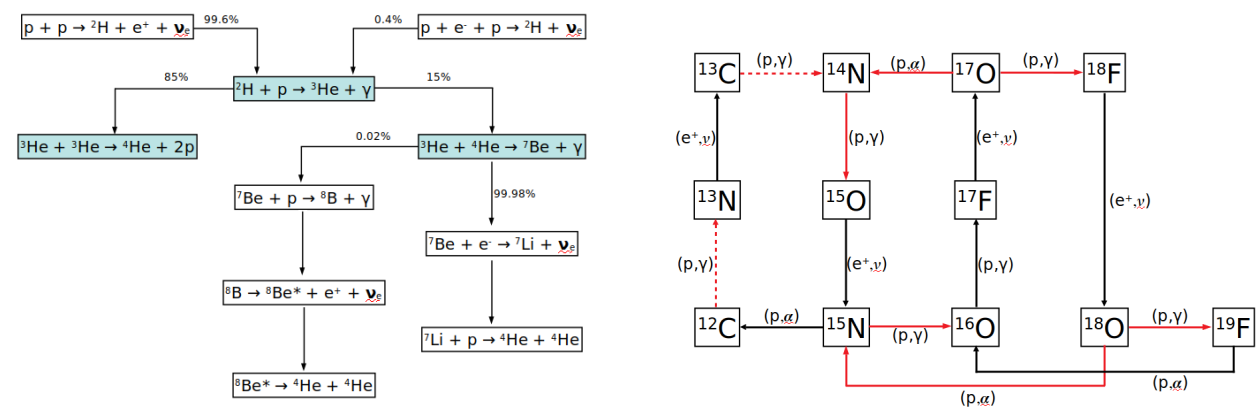

Fig. 2. Schematic drawing of the pp chain (left) and CNO cycle (right). Reactions of the pp chain investigated at LUNA are highlighted in light blue. CNO cycle reactions measured at LUNA are marked with solid red lines, while reactions that will be investigated in the future are marked with dashed red lines. 


\section{R. Depalo}

cycle involved in the production of oxygen isotopes in nova and AGB stars are ${ }^{17} \mathrm{O}(\mathrm{p}, \gamma){ }^{18} \mathrm{~F}$ and ${ }^{17} \mathrm{O}(\mathrm{p}, \alpha){ }^{14} \mathrm{~N}$ : Their ratio determines the leak rate from the second to the third CNO cycle (see Fig. 2) and it affects the synthesis of ${ }^{17,18} \mathrm{O}$ and ${ }^{18,19} \mathrm{~F}$. Moreover, the flux of $511 \mathrm{keV}$ gamma rays from classical novae, due to $\beta^{+}$decay of ${ }^{18} \mathrm{~F}$, strongly depends on the rate of ${ }^{17} \mathrm{O}(\mathrm{p}, \gamma){ }^{18} \mathrm{~F}$ and ${ }^{17} \mathrm{O}(\mathrm{p}, \alpha){ }^{14} \mathrm{~N}$.

At astrophysical energies the ${ }^{17} \mathrm{O}(\mathrm{p}, \gamma){ }^{18} \mathrm{~F}$ cross section depends on the narrow resonances at $64.5 \mathrm{keV}$ and $183 \mathrm{keV}$, lying on the tail of two broad resonances at 557 $\mathrm{keV}$ and $677 \mathrm{keV}$ and on the direct capture component. The $183 \mathrm{keV}$ resonance and the direct capture cross section have both been studied before LUNA by prompt gamma ray spectroscopy and detecting the $511 \mathrm{keV}$ gammas due to the annihilation of $\beta^{+}$particles from the decay of ${ }^{18} \mathrm{~F}$ (activation method) ${ }^{13}$.

The ${ }^{17} \mathrm{O}(\mathrm{p}, \alpha)^{14} \mathrm{~N}$ reaction was also investigated at LUNA delivering the proton beam to solid $\mathrm{Ta}_{2} \mathrm{O}_{5}$ targets enriched in ${ }^{17} \mathrm{O}$. Alpha particles from the reaction were detected with an array of Si detectors with an overall efficiency of the $10 \%$ and surrounded by a $5 \mathrm{~cm}$ thick lead shielding ${ }^{14}$. Alpha particle detectors running underground showed a background reduction of a factor of about 15 compared to overground $^{14}$. Thanks to this, both the $183 \mathrm{keV}$ resonance and the eak resonance at $64.5 \mathrm{keV}$ could be properly measured by LUNA in ${ }^{17} \mathrm{O}(\mathrm{p}, \alpha){ }^{14} \mathrm{~N}^{15}$.

For the $183 \mathrm{keV}$ resonance an $\omega \gamma=\left(1.68 \pm 0.03_{\text {stat }} \pm 0.12_{\text {syst }}\right) \mathrm{meV}$ was found, in good agreement with the literature.

For the $64.5 \mathrm{keV}$ resonance, the NACRE compilation gave a strength of $\omega \gamma=$ $5.5_{-1.5}^{+1.8} \mathrm{neV}^{16}$. A strength $\omega \gamma=\left(10.0 \pm 1.4_{\text {stat }} \pm 0.7_{\text {syst }}\right)$ neV was measured ${ }^{15}$. Such a value is about a factor of 2 higher than the previously estimated, thus leading to a factor of 2 increase in the reaction rate of shell hydrogen burning in red giant and AGB stars (see Fig. 2).

The new resonance strength has increased by $20 \%$ the predicted ${ }^{16} \mathrm{O} /{ }^{17} \mathrm{O}$ ratio after the first dredge up in red giant $\operatorname{stars}^{17}$ and it has allowed for the firm identification of the production site of a oxygen rich star grain population ${ }^{18}$.

\subsection{The ${ }^{22} \mathrm{Ne}(p, \gamma)^{23} \mathrm{Na}$ reaction}

The neon-sodium cycle of hydrogen burning contributes to the synthesis of the isotopes between ${ }^{20} \mathrm{Ne}$ and ${ }^{23} \mathrm{Na}$ in Red Giant Branch (RGB) stars ${ }^{19}$, Asymptotic Giant Branch (AGB) stars $^{20}$ and classical novae explosions ${ }^{21}$.

The ${ }^{22} \mathrm{Ne}(\mathrm{p}, \gamma)^{23} \mathrm{Na}$ Gamow window for AGB stars and classical novae extends from 50 to $600 \mathrm{keV}$. In this energy range, the proton capture on ${ }^{22} \mathrm{Ne}$ is dominated by a large number of resonances. While the resonances above $400 \mathrm{keV}$ have been recently measured with good precision ${ }^{22}$, none of the resonances below $436 \mathrm{keV}$ was ever observed in either direct or indirect experiments and only upper limits existed for their strengths ${ }^{16,23}$. Moreover, the mere existence of three resonances at 71,105 and $215 \mathrm{keV}$ was uncertain since it was tentatively reported $\mathrm{in}^{24}$ but never observed in subsequent experiments ${ }^{25,26}$. 
As a consequence, the ${ }^{22} \mathrm{Ne}(\mathrm{p}, \gamma)^{23} \mathrm{Na}$ reaction rates reported in the widely adopted compilations by $\mathrm{NACRE}^{16}$ and $\mathrm{C}$. Iliadis et al. ${ }^{23}$ are three orders of magnitude discrepant in the energy range of interest for hydrogen burning in AGB stars.

For this experimental campaign, a proton beam was delivered to the windowless gas target filled with $99.9 \%$ enriched ${ }^{22} \mathrm{Ne}$.

The gamma-rays emitted in the de-excitation of ${ }^{23} \mathrm{Na}$ were detected by two HPGe detectors collimated at $55^{\circ}$ (where the second order Legendre polynomial is zero and possible angular distribution effects are minimal) and $90^{\circ}$ with respect to the beam direction. The use of two detectors looking at different angles allows to estimate the effect of the gamma ray angular distribution on the resonance strength. In order to reduce the environmental background at gamma ray energies below 2.6 $\mathrm{MeV}$, the two detectors were surrounded by a copper and lead shielding, ensuring about four orders of magnitude background reduction ${ }^{27}$.

During about five months of data taking, all the resonances between 70 and 334 $\mathrm{keV}$ have been investigated. The resonances at 158, 186 and $259 \mathrm{keV}$ have been observed for the first time in a direct experiment. For these resonances, the complete excitation function was measured and then a long run at the energy of maximum yield was performed. New gamma decay modes have also been observed for the three resonances detected. For the non-detected resonances new upper limits have been measured. The new upper limits are two to three orders of magnitude lower than the previous direct measurement, proving the improvement in sensitivity that can be achieved in underground experiments ${ }^{28,29}$.

Fig. 3 shows the updated reaction rate for the ${ }^{22} \mathrm{Ne}(\mathrm{p}, \gamma)^{23} \mathrm{Na}$ reaction, calculated including the new LUNA results. Literature reaction rates adopted in references ${ }^{16}$ and ${ }^{23}$ are also shown for comparison.

Once the experiment with two HPGe detectors was completed, the ${ }^{22} \mathrm{Ne}(\mathrm{p}, \gamma){ }^{23} \mathrm{Na}$ reaction was investigated surrounding the gas target with a $4 \pi$ BGO detector. While the HPGe phase made it possible to study in great detail the decay
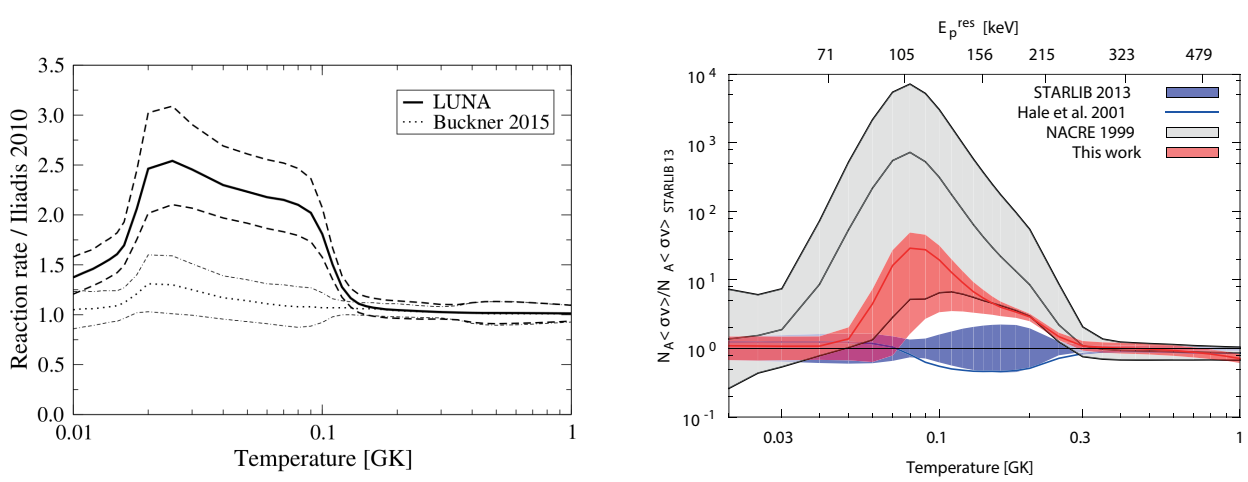

Fig. 3. Reaction rates obtained adopting the LUNA results on the ${ }^{17} \mathrm{O}(\mathrm{p}, \alpha)^{14} \mathrm{~N}$ (left) and the ${ }^{22} \mathrm{Ne}(\mathrm{p}, \gamma){ }^{23} \mathrm{Na}$ (right) reaction 


\section{R. Depalo}

modes of the resonances observed, the BGO phase allowed to increase the sensitivity to possible weak resonances by two orders of magnitude and to measure for the first time the direct capture contribution to the cross section directly at Gamow energies.

\subsection{Ongoing experiments}

A rich scientific program is underway at LUNA. Experiments for which the data analysis is being finalized include the ${ }^{22} \mathrm{Ne}(\mathrm{p}, \gamma)^{23} \mathrm{Na}$ BGO-phase, the study of the ${ }^{23} \mathrm{Na}(\mathrm{p}, \gamma){ }^{24} \mathrm{Mg}$ with both a germanium and a BGO detector, the ${ }^{18} \mathrm{O}(\mathrm{p}, \gamma){ }^{19} \mathrm{~F}$ and ${ }^{18} \mathrm{O}(\mathrm{p}, \alpha){ }^{15} \mathrm{~N}$ reactions.

Two reactions for which the data taking was just concluded are ${ }^{2} \mathrm{H}(\mathrm{p}, \gamma)^{3} \mathrm{He}$ and ${ }^{6} \mathrm{Li}(\mathrm{p}, \gamma){ }^{7} \mathrm{Be}$.

The ${ }^{2} \mathrm{H}(\mathrm{p}, \gamma)^{3} \mathrm{He}$ reaction takes part in the Big Bang Nucleosynthesis (BBN) network and it strongly influences the abundance of primordial deuterium. Observational information on the primordial deuterium abundance is obtained from the spectroscopic measurement of metal-poor damped Lyman-alpha (LDA) systems. These systems are the oldest astrophysical environments where deuterium is detected. A value of $[\mathrm{D} / \mathrm{H}]=(2.53 \pm 0.04) \cdot 10^{-5}$ has been recently obtained ${ }^{30}$ by averaging the best six astronomical measurements. This value is in fair agreement with the $[\mathrm{D} / \mathrm{H}]=(2.65 \pm 0.07) \cdot 10^{-5}$ predicted by the $\mathrm{BBN}$, although deuterium is the only primordial isotope with a smaller uncertainty on the measured than on the predicted abundance. The dominant source of uncertainty on the deuterium abundance is the cross section of ${ }^{2} \mathrm{H}(\mathrm{p}, \gamma)^{3} \mathrm{He}$ reaction. As a matter of fact, only a few experimental points exist in the energy region of interest with an overall systematic uncertainty at the $6-10 \%$ level $^{31}$. A higher ${ }^{2} \mathrm{H}(\mathrm{p}, \gamma)^{3} \mathrm{He}$ cross section in the $30-300$ $\mathrm{keV}$ energy range would improve the agreement between observed and predicted deuterium abundance andf recent ab initio calculations of the cross section have exactly provided such an enhancement ${ }^{32}$.

The cross section of the ${ }^{2} \mathrm{H}(\mathrm{p}, \gamma)^{3} \mathrm{He}$ reaction has already been measured at LUNA with the $50 \mathrm{kV}$ accelerator to explore the energy region where the reaction takes place in pre-main sequence stars and in the Sun. A new experiment has now been performed at the $400 \mathrm{kV}$ accelerator to cover the energy range of BBN. The experiment was performed with the windowless gas targets filled with deuterium at 0.3 mbar constant pressure, surrounded by two different types of gamma detectors, each in a different phase of the data taking: a $4 \pi$ BGO and high resolution HPGe. The high efficiency of the BGO $(\sim 70 \%)$ reduces the dependence of the detector response on the angular distribution of the emitted gamma ray. On the other hand, during the second phase of the experiment the angular distribution will be inferred by exploiting the high energy resolution of the germanium detector and the Doppler shift of gamma ray energies. 
The goal of the experiment is to reach an overall systematic uncertainty on the $3 \%$ level combining the results from the two phases.

The ${ }^{6} \mathrm{Li}(\mathrm{p}, \gamma){ }^{7} \mathrm{Be}$ reaction, involved in ${ }^{6} \mathrm{Li}$ depletion in pre-main sequence stars and in the BBN network, has also been investigated recently at LUNA. In stars, the ${ }^{6} \mathrm{Li}$ depletion proceedes mainly through the ${ }^{6} \mathrm{Li}(\mathrm{p}, \alpha){ }^{3} \mathrm{He}$ reaction. This reaction has been studied by many groups, and in order to explain the angular distribution of the emitted alpha particles, an R-matrix fit of the experimental data requires the contribution of both negative and positive parity excited states ${ }^{33}$. The existence of positive parity excited states in ${ }^{7} \mathrm{Be}$ is predicted by shell-model calculations, but has never been verified experimentally.

A recent measurement of the ${ }^{6} \operatorname{Li}(\mathrm{p}, \gamma){ }^{7} \mathrm{Be}$ cross section discovered a resonancelike structure around $195 \mathrm{keV}^{34}$. The observed S-factor is roughly reproduced by an R-matrix fit assuming the existence of an excited state with $\mathrm{E} \sim 5800 \mathrm{keV}, \mathrm{J}^{\pi}$ $=\left(1 / 2^{+}, 3 / 2^{+}\right)$and $\Gamma_{P} \sim 50 \mathrm{keV}$.

In order to verify the existence of such state, an experiment has been performed at LUNA using solid lithium targets of three different compositions (lithium oxide, lithium tungstate and lithium chloride), a HPGe detector mounted in close geometry and a Si detector to simultaneously measure alpha particles from the ${ }^{6} \mathrm{Li}(\mathrm{p}, \alpha){ }^{3} \mathrm{He}$ reaction. The data taking was completed in September 2017 and the analysis is presently ongoing.

A rich program is still scheduled for the LUNA $400 \mathrm{kV}$ accelerator. Other reactions that will be investigated in the next years include ${ }^{13} \mathrm{C}(\alpha, \mathrm{n}){ }^{16} \mathrm{O},{ }^{22} \mathrm{Ne}(\alpha, \gamma){ }^{26} \mathrm{Mg}$ and $(\mathrm{p}, \gamma)$ reactions on ${ }^{12} \mathrm{C}$ and ${ }^{13} \mathrm{C}$.

\section{The LUNA-MV project}

The future of LUNA involves the installation of a new higher voltage machine, in the MV range. This machine will be devoted to the study of nuclear reactions that take place at higher temperatures than those occurring during the hydrogen-burning processes studied so far with the $50 \mathrm{kV}$ and $400 \mathrm{KV}$ accelerator.

This project, named LUNA-MV, entails the realization of a unique facility inside the underground Gran Sasso Laboratory centered on a 3.5 MV single-ended accelerator providing high intensity hydrogen, helium and carbon beams ${ }^{6,7,8,9}$. Two different beam lines will be built, one devoted to solid target and the other to gas target experiments. This will allow the study of key reactions of helium burning (namely the ${ }^{12} \mathrm{C}(\alpha, \gamma){ }^{16} \mathrm{O}$ reaction and $(\alpha, \mathrm{n})$ reactions on ${ }^{12} \mathrm{C}$ and ${ }^{22} \mathrm{Ne}$ ) and to further investigate the ${ }^{14} \mathrm{~N}(\mathrm{p}, \gamma){ }^{15} \mathrm{O}$ and the ${ }^{3} \mathrm{He}\left({ }^{4} \mathrm{He}, \gamma\right){ }^{7}$ Be reactions, already measured at the LUNA $400 \mathrm{kV}$ facility, over a wide energy range in order to reduce their experimental uncertainties.

A key issue for LUNA-MV is the construction of a neutron shielding to preserve the low background characteristic of LNGS. In order to evaluate the neutron flux produced by the reactions above mentioned, a series of GEANT4 simulations of the 
LUNA-MV building was developed. The accelerator room will have thick concrete walls and ceiling working as neutron shielding to guarantee that the maximum neutron flux outside the building will be significantly smaller than the natural neutron background at LNGS, with a similar energy spectrum.

The construction of the infrastructure and the installation of the accelerator are scheduled in 2018.

\section{References}

1. C. E. Rolfs and W. S. Rodney, Cauldrons in the Cosmos, 1st edn. ( University Of Chicago Press, 1988).

2. A. Best et al., Eur. Phys. J. A 52, 72 (2016).

3. T. Szücs et al., Eur. Phys. J. A 44, 513 (2010).

4. U. Greife et al., Nucl. Inst. Meth. A 350, 327 (1994).

5. A. Formicola et al., Nucl. Inst. Meth. A 507, 609 (2003).

6. A. Guglielmetti, in Physics of the Dark Universe 4, 10 (2014).

7. LUNA Collaboration, Annual Report LNGS/EXP-02/16, Laboratori Nazionali del Gran Sasso 2015, pp. 142155.

8. M. Aliotta et al., The Eur. Phys. J. A 52, 76 (2016).

9. C. Broggini et al., Progr. Part. Nucl. Phys. 98, 55 (2018).

10. A. Formicola et al., Eur. Phys. J. A 52, 73 (2016).

11. A. Gustavino et al., Eur. Phys. J. A 52, 74 (2016).

12. A. Boeltzig et al., Eur. Phys. J. A 52, 75 (2016)

13. D.A. Scott et al., Phys. Rev. Lett. 109, 202501 (2012).

14. C.G. Bruno et al., The Eur. Phys. J. A 51, 94 (2015).

15. C.G. Bruno et al., Phys. Rev. Lett. 117, 142502 (2016).

16. C. Angulo et al., Nucl. Phys. A 656, 3 (1999).

17. O. Straniero et al., Astron. Astrophys. 598, A128 (2017).

18. M. Lugaro, et al., Nat. Astron. 1, 0027 (2017).

19. E. Carretta et al., Astron. Astrophys. 505, 117 (2009).

20. R.G. Izzard et al., Astron. Astrophys. 466, 641 (2007)

21. C. Iliadis et al., Astrophys. J. Suppl. 142, 105 (2002)

22. R. Depalo et al., Phys. Rev. C 92, 045807 (2015).

23. C. Iliadis et al., Nucl. Phys. A 841, 1 (2010)

24. J.R. Powers et al., Phys. Rev. C 4, 6 (1971).

25. S.E. Hale et al., Phys. Rev. C 65, 015801 (2001).

26. D.G. Jenkins et al.Phys. Rev. C 87, 064301 (2013).

27. F. Cavanna et al.,Eur. Phys. J. A 50, 179 (2014).

28. F. Cavanna et al., Phys. Rev. Lett. 115, 252501 (2015).

29. R. Depalo et al., Phys. Rev. C 94, 055804 (2016).

30. R.J. Cooke et al., Astrophys. J. 781, 31 (2014).

31. E. Adelberger, et al., Rev. Modern Phys. 83, 195 (2011).

32. L.E. Marcucci et al., Phys. Rev. Lett. 116, 102501 (2016).

33. J. Cruz et al., J. Phys. G Nucl. Part. Phys. 35, 014004 (2008).

34. J. He et al., Phys. Lett. B 725, 287 (2013). 